\section{AB0123 T-CELL SURFACE GLYCOSYLATION PATTERN ALTERATIONS IN SLE - A PUTATIVE LINK TO GALECTIN-1-MEDIATED IMMUNOREGULATORY DEFICIENCY}

Á. Hornung ${ }^{1}$, E. Szabó ${ }^{2}$, Á. Czibula ${ }^{2}$, É. Monostori ${ }^{2}$, L. Kovács ${ }^{1} .{ }^{1}$ Department of Rheumatology and Immunology, University of Szeged; ${ }^{2}$ Department of

Genetics, Hungarian Academy of Sciences, Biological Research Centre, Szeged, Hungary

Background: We have previously found that activated T-cells from systemic lupus erythematosus (SLE) patients express lower amount of intracellular galectin-1 (icGal-1) than those of healthy controls and are resistant to the apoptotic effect of extracellular galectin-1 (ecGal-1), an endogenous immunoregulatory lectin. We also demonstrated that the de novo synthesized icGal-1 level affects apoptosis of T-cells induced by ecGal-1, since low icGal-1 expression resulted in reduced sentsitivity to ecGal-1 (Deák $\mathrm{M}$ et al). We have therefore proposed the defective icGal-1 production to be an explanation to the insufficient regulatory effects of ecGal-1 in SLE. However, altered binding of ecGal-1 to T-cells due to changes in surface glycosylation may also regulate the apoptotic activity of ecGal-1.

Objectives: We have herein hypothesized that the cell-surface glycosylation pattern, and consequently, lectin-binding ability in SLE T-cells is altered, and that an abnormal expression of glycosylation enzymes may account for these changes.

Methods: In order to analyse the glycosylation pattern of cell surface glycoproteins, lectin-binding assays were performed using 5 different plant lectins and human recombinant Gal-1 on resting and activated T-cells from patients with active SLE $(n=8)$ with multi-colour flow-cytometry, and were compared with 15 healthy controls. mRNA levels of 13 glycosylation enzymes involved in the development of $\mathrm{N}$-glycan structures on T-cells were measured with $\mathrm{qPCR}$, and were correlated with the specific lectin binding data.

Results: As compared with the resting state, the increase in Gal-1 binding during activation was significantly lower in SLE T-cells than in controls, and the level of Gal-1 binding maximum was significantly reduced in SLE activated T-cells than in controls. Binding maximum of plant lectins that recognise high complexity $\mathrm{N}$-glycans also increased less in SLE T-cells than in controls during activation. mRNA level of sialyltransferase ST3GAL6 was increased and neuraminidase Neu1 was decreased in active SLE patients as compared to controls. The ST6GAL1/NEU1 ratio in SLE patients positively correlated with the SLEDAI disease activity index.

Conclusions: SLE T-cells show decreased complexity of $\mathrm{N}$-glycan structures. Increased ST3GAL6 and decreased Neu1 expression result in an increased density of terminal sialic acids, and this may explain the impaired Gal-1 binding. In addition to the previously described deficiency in icGal-1 expression upon activation, our present findings of an attenuated glycan complexity and a shift toward terminal sialylation provide a further mechanism of pathological T-cell activation and regulation of T-cell viability in SLE.

References:

[1] Deák M, Hornung Á, Novák J, Demydenko D, Szabó E, Czibula Á, FajkaBoja R, Kriston-Pál É, Monostori É, Kovács L. Novel role for galectin-1 in T-cells under physiological and pathological conditions. Immunobiology. 2015 Apr;220(4):483-9.

Disclosure of Interest: None declared

DOI: 10.1136/annrheumdis-2017-eular.5657

\section{AB0124 SEROLOGICAL MEASURES OF B CELL FUNCTION IN PATIENTS WITH SLE; HOW ROBUST ARE THEY OVER TIME?}

A. Hennessey ${ }^{1,2}$, R. Marques $^{3}$, M. Leandro ${ }^{1,2}$, D. Isenberg ${ }^{1,2}$, G. Cambridge ${ }^{2}$. ${ }^{1}$ Division of Rheumatology, Department of Medicine, University College London Hospitals; ${ }^{2}$ Centre for Rheumatology and Bloomsbury Rheumatology Unit, University College London, London, United Kingdom; ${ }^{3}$ Serviço de Medicina Interna B, Centro Hospitalar Universitário de Coimbra, Coimbra, Portugal

Background: In SLE the precise mechanisms whereby parent autoreactive $B$ cells are generated and permitted to escape tolerance checkpoints, proliferate, persist and switch to pathogenic autoantibody production remains poorly understood. Hypotheses include defective negative central selection, defective peripheral selection with enhanced germinal center activity as well as positive selection of $\mathrm{B}$ cells via autoantigen presentation or T-independent mechanisms. Biomarkers to identify possible breaches in tolerance checkpoints would allow more effective intervention. We have therefore measured relative levels of soluble (s)CD23 (released during B cell differentiation from naive to memory B cell status) and the B cell activation factor, BAFF (survival factor and class-switch/differentiation promoter) in SLE sera to determine if relative levels of SCD23 and BAFF were of use as biomarkers to group patients based on $B$ cell kinetics rather than clinical features. BAFF is often raised in SLE patients and can stimulate the aberrant differentiation of transitional $B$ cells and of plasmablasts in vitro. Combining the 2 biomarkers could therefore indicate whether there was increased expansion of naïve B cells, and whether BAFF was a possible driver/consequence of autoimmunity in different patients.

Objectives: To determine whether relative levels of $\mathrm{SCD} 23$ and BAFF reflect disease activity or remain stable over time.

Methods: Stored SLE serum from patients who were Rituximab-naive, had $>5$ samples available in the biobank over at least 6 months $(n=38)$. Samples were analysed for levels of SCD23 and BAFF via ELISA. Wilcoxon matched-pairs signed rank test were used to compare serial values. Clinical details including BILAG scores were also collected for the available time points. If positive dsDNA at any time during the time period this was deemed positive. The latest BILAG was utilised and reviewed, a patient was deemed not to have system involvement if they had an "E" for that system.

Results: Sera from 38 SLE patients (32 female, 6 male) with mean age of first sample 42 (range: $25-72$ ). Minimum interval between 1st and last sample was 101 weeks (range: $35-192$ weeks). Patients were then sorted into clinical groups according to levels of these serum markers. Normal ranges defined as: SCD23 (1235-5023pg/ml), BAFF (584-1186pg/ml). Group I ( $n=9)$ : - High (H) sCD23, Normal (N) BAFF; Group II $(n=11)-\mathrm{H}$ sCD23, H BAFF; Group III $(\mathrm{n}=17)-\mathrm{N}$ sCD23, N BAFF; Group IV $(n=2)-N$ sCD23, H BAFF. Figure 1 shows the $p$ values generated from the Wilcoxon matched-pairs signed rank test for each time period demonstrating nil significant change over time. Analysis of clinical data showed no differences in terms of organ involvement or anti-dsDNA -Ab status.

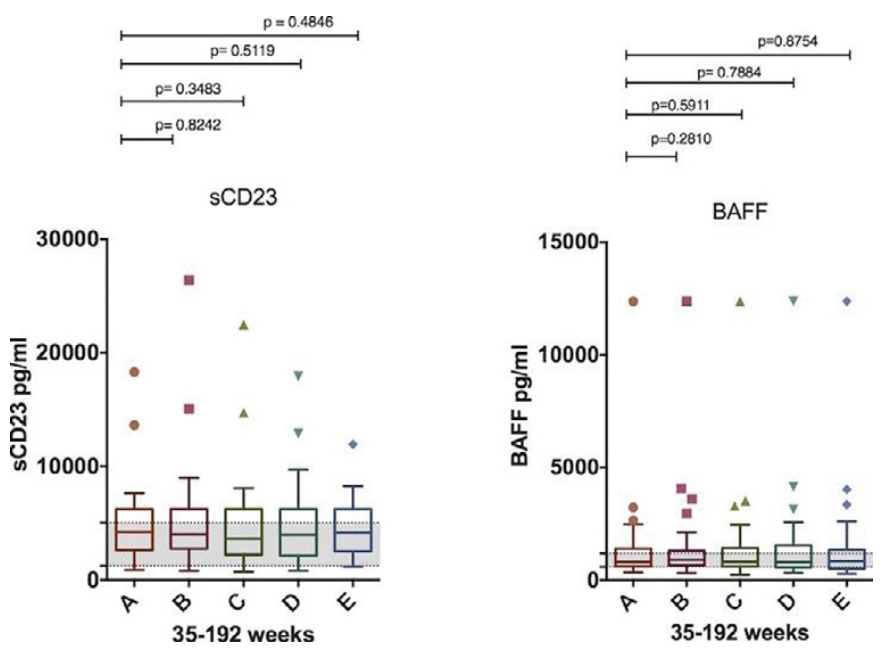

Conclusions: Within a cohort of SLE patients, soluble CD23 and BAFF is stable over time despite variation in disease activity. Grouping patients based on SCD23 and BAFF profile may be useful in identifying distinct B cell maturation pathways reflecting underlying autoimmune pathways which vary between patients.

Disclosure of Interest: A. Hennessey: None declared, R. Marques: None declared, M. Leandro Consultant for: Roche UK, Roche Basel and Genentech, D. Isenberg: None declared, G. Cambridge: None declared DOI: 10.1136/annrheumdis-2017-eular.6780

\section{AB0125 BRAIN IMMUNOPATHOLOGY OF LUPUS-PRONE FCIRIIB-/-YAA MICE - IMPLICATION TO THE INNATE IMMUNE RELATED MECHANISM OF NEUROPSYCHIATRIC SLE}

A. Nomura ${ }^{1}$, G. Murayama ${ }^{1,2}$, A. Chiba ${ }^{1}$, S. Miyake ${ }^{1} .{ }^{1} /$ mmunology; ${ }^{2}$ Rheumatology, Juntendo University, Tokyo, Japan

Background: Neuropsychiatric SLE (NPSLE) is a common manifestation of SLE and problems such as cognitive impairment or depression are elusive. The importance of innate immune related inflammation in the pathomechanism of neurodegenerative or psychiatric diseases has been recognized recently, and the importance of innate immunity in the pathogenesis of NPSLE has also been suggested ${ }^{1}$. Therefore, we investigated innate immune mechanism of NPSLE by using lupus prone mice.

Objectives: This study is conducted to understand the brain immune pathology of lupus-prone $\mathrm{Fc} \gamma \mathrm{RIIB}^{-/-}$Yaa mice in which innate immune stimulation is potentiated by the duplication of Toll-like receptor $7^{2}$.

Methods: Immune cell subsets and histopathology of brains were analyzed by flow cytometry and immunohistochemistry in $\mathrm{Fc}_{\mathrm{R}} \mathrm{RIIB}^{-/}$Yaa mice compared with congenic mice at around 16 week-old when glomerulonephritis had developed. For flow cytometric analysis, microglia, myeloid lineage cells and lymphocytes were defined by staining with CD11b and CD45. Subsets of those cells and their activation status were analyzed. For histopathological analysis, microglia, brain macrophages, astrocytes and lymphocytes were immunostained and expression of $\mathrm{MHC}$ class I and class II were also analyzed.

Results: Flow cytometric analysis revealed increase in the number of microglial cells (CD11 b $\left.\mathrm{b}^{+} \mathrm{CD} 45^{\text {int }}\right)$ and myeloid lineage cells (CD11b+ $\left.\mathrm{b}^{+} \mathrm{CD} 45^{\text {high }}\right)$ in the brains of $\mathrm{Fc} \gamma \mathrm{RIIB}^{-/}$Yaa mice compared with congenic $\mathrm{Fc} \gamma \mathrm{RIIB} \mathrm{B}^{+/+}$mice. Mean fluorescence intensity of MHC class I was increased in microglia and myeloid lineage cells in $\mathrm{Fc}_{\mathrm{C}} \mathrm{RIIB}^{-/}$Yaa mice. An increased percentage of CD3 positive cells compared to CD19 positive cells were observed and their expression of CD69, an activation marker, were increased in $\mathrm{Fc} \mathrm{RIIB}^{-1-}$ Yaa mice. In histopathology, number of macrophages and microglia identified by lba1 (Ionized calcium binding adapter molecule 1) positive cells were increased in $\mathrm{Fc}_{\gamma} \mathrm{RIIB}^{-/}$Yaa mice. In areas where MHC class I and class II were highly expressed on macrophages, 